\title{
Remote information concentration using a bound entangled state
}

\author{
Mio Murao ${ }^{1}$ and Vlatko Vedral ${ }^{2}$ \\ ${ }^{1}$ Semiconductors Laboratory, The Institute of Physical and Chemical Research (RIKEN), Wako-shi 351-0198, Japan \\ ${ }^{2}$ Centre for Quantum Computation, Clarendon Laboratory, University of Oxford, Oxford OX1 3PU, United Kingdom
}

(October 23, 2018)

\begin{abstract}
Remote information concentration, the reverse process of quantum telecloning, is presented. In this scheme, quantum information originally from a single qubit, but now distributed into three spatially separated qubits, is remotely concentrated back to a single qubit via an initially shared entangled state without performing any global operations. This entangled state is an unlockable bound entangled state and we analyze its properties.
\end{abstract}

PACS numbers: 03.67.Hk, 03.67.-a

Quantum entanglement has generated a great deal of interest recently as the most important resource in quantum information processing. The protocols of super dense coding [1], quantum teleportation [2] and telecloning 3] cannot be performed without some form of entanglement between the parties involved: classical correlations alone can never achieve the quantum efficiency arising from entanglement. Given that entanglement is a resource, it is important to be able to quantify it in order to deduce how effectively we can process information. There have been a number of suggestions for quantifying entanglement [4], but the most fruitful method comes from a procedure known as entanglement distillation [5]. In this procedure, two distant users, Alice and Bob, share a certain number of entangled pairs all in the same state $\rho$. They then are allowed to perform local operations and communicate classically with each other (LOCC). The question is how many maximally entangled pairs they can obtain in this way. The limit of distillation in the infinite number of initial copies of $\rho$ is known as the entanglement of distillation [5]. A natural question to ask is: which states $\rho$ can be distilled to maximally entangled states? Separable states $\rho=\sum_{i} \rho_{A}^{i} \otimes \rho_{B}^{i}$ are clearly non-distillable. Surprisingly, however, a recent important discovery by the Horodecki family showed that there are also some entangled states which cannot be distilled [6]. These states have appropriately been called bound entangled. They are peculiar as entanglement has to be invested in creating them by LOCC, but this invested entanglement cannot then be recovered by LOCC. Bound entanglement has been studied extensively in the last two years [7], nevertheless no information processing protocol has been found where bound entangled states perform better than just classically correlated states. Therefore it has seemed that they are useless for quantum information processing and that we always need to use some form of "free" (unbound) entanglement to achieve greater-thanclassical efficiency. However, as we show in this letter, this is not the case.

We present an important protocol where bound en- tanglement can be utilized effectively and performs better than any classically correlated states. This protocol is remote information concentration, the inverse of telecloning [3]. Quantum telecloning, as its name suggests, combines teleportation and cloning in such a way that a sender teleports an unknown qubit state $|\phi\rangle=\alpha|0\rangle+\beta|1\rangle$ to a number of spatially separated receivers simultaneously. These teleported qubits cannot, of course, be exact replicas of the original qubit due to the linear laws of quantum evolution ("no-cloning theorem") [8]. However it has been shown that fidelities as high as allowed by the non-exact cloning (known as optimal cloning [9]) can be achieved. The optimal cloning state for $|\phi\rangle$ is represented by a three qubit state

$$
\begin{aligned}
\left|\psi_{c}\right\rangle & =\alpha \sqrt{\frac{2}{3}}\left\{|0\rangle|00\rangle+\frac{1}{2}|1\rangle(|01\rangle+|10\rangle)\right\} \\
& +\beta \sqrt{\frac{2}{3}}\left\{|1\rangle|11\rangle+\frac{1}{2}|0\rangle(|01\rangle+|10\rangle)\right\} .
\end{aligned}
$$

where the first qubit is an ancilla and the last two qubits are two optimal clones. Now the question we ask is: once a state has been telecloned to spatially separated parties, can it then be recreated using only LOCC? The answer is yes and surprisingly involves a recently constructed unlockable bound entangled state $[10]$.

The four particle unlockable bound entangled state presented by Smolin 10] is

$$
\rho_{u b}=\frac{1}{4} \sum_{i=0}^{3}\left|\Phi^{i}\right\rangle\left\langle\Phi^{i}|\otimes| \Phi^{i}\right\rangle\left\langle\Phi^{i}\right|
$$

where $\left|\Phi^{i}\right\rangle$ represents the four Bell states, $\left|\Phi^{0}\right\rangle=$ $(|00\rangle+|11\rangle) / \sqrt{2}, \quad\left|\Phi^{1}\right\rangle=(|00\rangle-|11\rangle) / \sqrt{2}, \quad\left|\Phi^{2}\right\rangle=$ $(|01\rangle+|10\rangle) / \sqrt{2}$ and $\left|\Phi^{3}\right\rangle=(|01\rangle-|10\rangle) / \sqrt{2}$. This state is not distillable if we do not allow joint quantum operations (i.e. if all four parties only operate locally), and is therefore, a bound entangle state. However, if we allow a two qubit joint operation, i.e. Bell joint measurement on any two qubits, we can obtain a maximally 
entangled state for the other two qubits via LOCC. Thus this state is unlockable. The unlocking mechanism is based on a joint operation for two out of four qubits.

Before we explain remote information concentration, we briefly summarize the forward process, telecloning. We focus on the 1 to 2 telecloning and its reverse in this letter. Generalizations to more qubits are possible and will be investigated elsewhere. The telecloning scheme [3] allows direct distribution of optimal clones from an single original qubit state $|\phi\rangle$ to spatially separated parties using LOCC. In the telecloning scheme, we use an initially shared entangled channel (telecloning state)

$$
\begin{aligned}
\left|\xi_{t c}\right\rangle & =\frac{1}{\sqrt{3}}\{|00\rangle|00\rangle+|11\rangle|11\rangle \\
& \left.+\frac{1}{2}(|01\rangle+|01\rangle)(|01\rangle+|01\rangle)\right\} .
\end{aligned}
$$

where the first qubit is an input port of the distributor, the second qubit is an output port for the ancilla, and the third and forth qubits are output ports for the optimal clones. The telecloning protocol [3] is similar to teleportation; the distributor performs a Bell joint measurement between the unknown state and the input port qubit, and then the receivers, who hold output port qubits, perform a single qubit operations depending on the distributor's measurement result.

Now we present our remote information concentration scheme. From the distributed optimal cloning qubits shared by the spatially separated parties (Alice who holds the ancilla qubit and Bob and Charlie who each hold a clone qubit), the original single qubit state is recreated at the location of an receiver, David in our scheme: $\left|\psi_{c}\right\rangle_{A B C} \rightarrow|\phi\rangle_{D}$. We employ the unlockable bound entangled state (Eq.(2)) as an entangled channel for this scheme. The four qubits of the unlockable bound entangled state are initially distributed to Alice, Bob and Charlie (input port qubits) and David (output port qubit). The three senders, Alice, Bob and Charlie, individually perform Bell joint measurements between their qubits of the optimal cloning state and their input port qubits. We stress that no global operation is allowed between qubits belonging to different parties. One of the four possible outcomes $\left\{\Phi^{i}\right\}$ is obtained by the measurement of a party. All three senders classically communicate their measurement results with David. $(2 \times 3=6$ bits of classical information is communicated in total.) Each Bell measurement result $\left\{\Phi^{i}\right\}$ is associated with the corresponding Pauli operators $\left\{\sigma_{i}\right\}$, where $\sigma_{0} \equiv \mathbf{1}$, $\sigma_{1} \equiv \sigma_{z}, \sigma_{2} \equiv \sigma_{x}$, and $\sigma_{3} \equiv \sigma_{z} \cdot \sigma_{x}$. David performs a Pauli operation $\sigma_{j}$, which is the product (up to a global phase factor) of the three Pauli operators associated with the three Bell measurements, on his output port qubit. The output port of David is now in the original state $|\phi\rangle$. A schematic picture of this protocol is shown in Fig.1. Since we do not allow joint operations on spatially separated qubits, the information channel in our scheme is indeed bound entangled. It is surprising that a bound entangled state can actually be useful for "transmitting" quantum information. In the following, we analyze this feature from two points of view: remote quantum operation and entanglement structure.

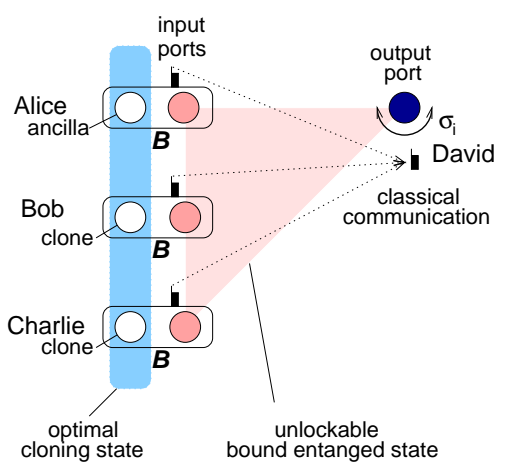

FIG. 1. Schematic picture showing the concentration of information from Alice, Bob and Charlie at the remote receiver, David, using an unlockable bound entangled state.

Remote quantum operation is performance of (global) unitary operations on remote qubits: A unitary operation $U$ is implemented by an initially shared entangled channel, Bell measurement, classical communication and (simple) single qubit operations, instead of directly running a quantum circuit. This is a generalization of quantum teleportation [2]. Telecloning [3] and quantum information distribution via entanglement [11] are examples of remote quantum operation for $1 \rightarrow N$ quantum optimal cloning with $d$-level particles, which requires one input port and $2 N-1$ output ports. More general cases requiring more than one input port have been studied by Gottesman and Chuang [12] in the context of "quantum computation using teleportation" and "quantum software". The initially shared entanglement in the remote quantum operation scheme functions as quantum software. According to their result, unitary operations which belong to the Clifford group 13 can be implemented remotely, if we restrict the single qubit operations to be the Pauli operations. The compounding qubits of the shared entanglement need not be in the same location. In this case, the share entanglement functions as a transmission channel as well as quantum software. We consider this most restricted case of all-separated qubits.

To implement a unitary operation $U$ on a state of three input qubits $(|\psi\rangle)$, the entangled channel consists of three input port qubits and three output port qubits. For unitary operations that can be decomposed into CNOT (controlled NOT) and Hadamard gates, which are members of the Clifford group [13], the entangled channel state is given by

$$
|\xi\rangle=\sum_{\tilde{k}=\tilde{0}}^{\tilde{7}}|\tilde{k}\rangle \otimes U|\tilde{k}\rangle,
$$

where $\tilde{k}$ is a 3 -bit binary number, for example, $\tilde{0}=000$, 
$\tilde{1}=001, \ldots, \tilde{7}=111$. The first three qubits are the input ports and the last three qubits are the output ports. All the qubits of this channel are spatially separated from each other. We assume that Alice, Bob, Charlie, David, Elizabeth, Fred each hold one qubit of the channel (in this order). Alice, Bob and Charlie individually perform Bell joint measurements on their input qubits (in the state $|\psi\rangle_{A B C}$, the qubits to be processed) and the input port qubits. David, Elizabeth and Fred perform an appropriate Pauli operation depending on the measurement results of Alice, Bob and Charlie. The mapping between measurement results and Pauli operations is initially agreed. The final state of David, Elizabeth and Fred is $U|\psi\rangle_{D E F}$.

Now we return to reverse optimal cloning. We define a reverse cloning unitary operator $U_{r}$

$$
U_{r}\left|\psi_{c}\right\rangle=|\phi\rangle \otimes \sqrt{\frac{2}{3}}\left(|00\rangle+\frac{|01\rangle+|10\rangle}{2}\right),
$$

where the last two qubits are ancillas that are disentangled from the first qubit which holds the concentrated single qubit information. Note that $U_{r}$ does not initialize the ancilla qubits after the operation into the conventional ancilla state $|00\rangle$. $U_{r}$ can be decomposed into just CNOT gates as shown in Fig.2. Thus the reverse cloning operation is in the Clifford group and can be performed by remote quantum operation. $U_{r}$ is explicitly given in the computational basis by

$$
U_{r}=\left(\begin{array}{llllllll}
1 & 0 & 0 & 0 & 0 & 0 & 0 & 0 \\
0 & 0 & 0 & 0 & 0 & 1 & 0 & 0 \\
0 & 0 & 0 & 0 & 0 & 0 & 1 & 0 \\
0 & 0 & 0 & 1 & 0 & 0 & 0 & 0 \\
0 & 0 & 0 & 0 & 0 & 0 & 0 & 1 \\
0 & 0 & 1 & 0 & 0 & 0 & 0 & 0 \\
0 & 1 & 0 & 0 & 0 & 0 & 0 & 0 \\
0 & 0 & 0 & 0 & 1 & 0 & 0 & 0
\end{array}\right) .
$$

Inserting $U_{r}$ in Eq.(5), we obtain the channel for the remote reverse cloning:

$$
\begin{aligned}
\left|\xi_{r c}\right\rangle & =\frac{1}{2 \sqrt{2}}\{(|0000\rangle+|1111\rangle)|00\rangle+(|0101\rangle+|1010\rangle)|01\rangle \\
& +(|0011\rangle+|1100\rangle)|10\rangle+(|0110\rangle+|1001\rangle)|11\rangle\}
\end{aligned}
$$

In this expression, Alice, Bob, Charlie and David hold the first, second, third and fourth qubits, respectively. The last two qubits are ancillas and may be separated from the other qubits (their location is irrelevant). From Eq.(8), we obtain the unlockable bound entangled state $\rho_{u b}$, if we trace out the ancilla variables. Since the operations performed on ancillas do not effect the output port qubit, we can trace out the ancilla variables from the beginning. Then this remote quantum operation is equivalent to remote information concentration. This is why the unlockable bound entangled state actually functions as a channel for remote information concentration.

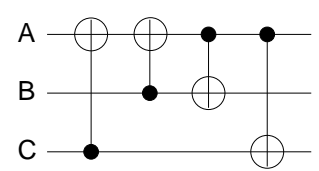

FIG. 2. Quantum circuit for the unitary operation of reverse cloning $U_{r}$. "A" represents an ancilla qubit, and "B" and "C" are the optimal clone qubits. The symbol "•" represents the control qubit and they symbol " $\oplus$ " represents the NOT operation

Next, we analyze the remote information concentration scheme from the view point of entanglement structure by considering the amount of entanglement in various separation cuts. First, we consider the pure state representation of the channel including the ancillas (Eq.(8)). For simplicity, we denote the six qubits as A, B, C, D, E, F. From Eq.(5), we see that the channel can be considered as a pair of maximally entangled 8-level particles, if we separate DEF from ABC. This means that there are 3ebits of entanglement across the cut ABC:DEF. This is why the information of three input qubits can be processed and faithfully transmitted via teleportation from the three senders to the three receivers. We now consider the entanglement structure of the unlockable bound entangled state. In our scheme, at least 1-ebit of entanglement is required across the $\mathrm{ABC}$ : $\mathrm{D}$ cut for the faithful transmission of a single qubit quantum information. We calculate the relative entropy of entanglement 14] across the ABC:D cut. Relative entropy of entanglement for a mixed state is defined by $E_{R E}=\min _{\sigma \in D} S(\rho \| \sigma)$, where $S(\rho \| \sigma)=\operatorname{Tr} \rho \log \rho-\rho \log \sigma$ is the quantum relative entropy, and the minimum is taken over $D$, the set of separable states. We can prove that in our case $E_{R E}=1$. The amount of entanglement across the ABC:D cut is indeed 1-ebit. However, as we have described before, there is no distillable pairwise entanglement in the unlockable bound entangled state, if no joint operations are allowed for qubits in different locations. Therefore the 1-ebit of entanglement across ABC:D does not explain the successful concentration of information. How can the information be processed and faithfully transmitted only by LOCC?

8) The answer lies in the optimal cloning state. The three qubits in the optimal cloning state are actually entangled each other. The Peres-Horodecki criterion [15], which is the smallest eigenvalue of partially transposed reduced density matrix of an ancilla qubit and a clone qubit $\rho_{12} \equiv \operatorname{tr}_{3}\left|\psi_{c}\right\rangle\left\langle\psi_{c}\right|$, is $(1-\sqrt{17}) / 12 \sim-0.26$. This negativity shows that each of two clone qubits is entangled (although not maximally entangled) with the ancilla qubits and this entanglement is not bound entanglement. We may conjecture how information processing and transmission have been achieved using only a bound entangled state and LOCC in our scheme as follows: the Bell joint measurement "combines" the optimal cloning state (the input state) and the bound entangled state (the channel 
state), which is initially "closed" for transmission. The unbound entanglement of the input state provides quantum correlation among the qubits of the bound entangled state. The quantum correlation "opens" the channel for transmitting concentrated single qubit information from distributed in three qubits of the input state. Entanglement of the input optimal cloning state and the unlockable bound entangled channel state function in a complementary fashion. This result explains the importance of the ancilla qubit in the optimal cloning state, since the ancilla qubit is necessary for holding entanglement.

Another interesting observation is that the unlockable bound entangled state is also valid for remotely concentrating information from the spatially separate 3 -qubit error correction state: $\left|\psi_{e}\right\rangle_{A B C}=\alpha|000\rangle+\beta|111\rangle \rightarrow$ $|\phi\rangle_{D}$. The procedure is similar to the case of optimal cloning. The only difference is a modification to the mapping to Pauli operations. David performs an additional $\sigma_{2}$ if the measurement results from Bob or Charlie, but not both, belong to the set $\left\{\left|\Phi^{0}\right\rangle,\left|\Phi^{1}\right\rangle\right\}$. In this case, we may again consider that the (unbound) entanglement of the input state $\left|\psi_{e}\right\rangle$ "opens" the bound entangled channel for transmitting concentrated single qubit information from the input state. If we consider the quantum state $|\phi\rangle$ as a quantum key [16], remote information concentration together with information distribution [1] may allow more secure distribution of the quantum key to David via spatially-separated, branched repeaters Alice, Bob and Charlie.

Finally, we show that no classically correlated state can achieve the same task (c.f. [4]). In optimal cloning scheme, due to the linearity of quantum transformations, mixed states can be cloned as well as pure. The same of course holds for telecloning. We consider the case when the qubit to be telecloned is maximally entangled with another qubit of George. After telecloning the qubit state into the qubits of Alice, Bob and Charlie, we perform the reverse process and remotely concentrate information at the location of David. Consequently, the qubits of David and George become entangled. If a shared state with only classical correlation could perform this remote information concentration, the procedure would create entanglement between David and George. This, however, is not possible: entanglement cannot be increased by LOCC. Therefore no classically correlated state can perform remote information concentration.

In this letter we have presented remote information concentration, the reverse process of quantum telecloning. It was shown that, surprisingly, the state needed for this operation is a bound entangled state. We have analyzed the remote information concentration scheme from two points of view, considering remote quantum operations and analyzing the entanglement structure of the bound state and the input state. We have shown that the unlockable bound entangled state is a reduced density matrix for the entanglement channel of remote reverse cloning, if we trace out the ancilla qubits of the output state. From our entanglement structure analysis, we have found that the functions of the entanglement of the optimal cloning state and the unlockable bound entangled state are complementary. We have also shown that the unlockable bound entangled state can be used for remotely concentrating information from a distributed 3qubit error correction state, which may be useful for secure transmission of a quantum key. Furthermore, we showed that no purely classically correlated state can achieve this task. We hope that our work would stimulate more research into the nature of entanglement and its general usefulness in quantum information processing.

The authors are grateful to J. Watson for his help during the preparation of this manuscript. M.M. is supported by the Special Postdoctoral Researchers Program of RIKEN, and the Grant-in-Aid for Encouragement of Young Scientists (Grant No. 12740253) by Japan Society of the Promotion of Science. V.V. gratefully acknowledges funding by the European Union project EQUIP (Contract No. IST-1999-11053) and Hewlett Packard. Parts of this work were completed during the Banasque Center for Science program "Quantum Information Processing" (2000).

[1] C. H. Bennett and S. J. Wiesner, Phys. Rev. Lett. 69, 2881 (1992).

[2] C.H. Bennett et al, Phys. Rev. Lett. 70, 1895 (1993).

[3] M. Murao et al, Phys. Rev. A 59, 156 (1999).

[4] M. B. Plenio and V. Vedral, Cont. Phys. 39431 (1998).

[5] C. H. Bennett et al, Phys. Rev. A 54, 3824 (1996).

[6] M. Horodecki, P. Horodecki, R. Horodecki, Phys. Rev. Lett. 80, 5239 (1998).

[7] M. Lewenstein et al, quant-ph/0006064.

[8] W. K. Wootters and W. H. Zurek, Nature 299, 802 (1981).

[9] V. Bužek and M. Hillery, Phys. Rev. A 54, 1844 (1996); M. Hillery and V. Bužek, Phys. Rev. A 561212 (1997); V. Bužek et al, Phys. Rev. A 56, 3446 (1997).

[10] J. Smolin, quant-ph/0001001.

[11] M. Murao, M.B. Plenio and V.Vedral, Phys. Rev. A 61, 042311 (2000).

[12] D. Gottesman and I.L. Chuang, Nature 402, 390 (1999).

[13] D. Gottesman, quant-ph/9807006.

[14] V. Vedral et al, Phys. Rev. Lett. 78, 2275 (1997); V. Vedral and M. B. Plenio, Phys. Rev. A 57, 1619 (1998).

[15] A. Peres, Phys. Rev. Lett. 77, 1413 (1996); M. Horodecki, P. Horodecki and R. Horodecki, Phys. Lett. A 223, 1 (1996).

[16] C.H. Bennett and G. Brasared, in Proc. IEEE Int. Conference on Computers, Systems and Signal Processing (IEEE, New York), 175 (1984). 